\title{
Comparative numerical study of single and two-phase models of nanofluid liquid film evaporation in a vertical channel
}

\author{
Monssif Najim ${ }^{1,2,{ }^{*}}$, M'barek Feddaoui ${ }^{1}$, Abderrahman Nait Alla ${ }^{1}$, and Adil Charef ${ }^{1}$ \\ ${ }^{1}$ Laboratory of Energy, Materials and Systems Engineering, Ibn Zohr University, Agadir, Morocco \\ ${ }^{2}$ Department of physics, Faculty of Science and Technology, University Hassan II, Mohammedia, Morocco
}

\begin{abstract}
The main purpose of this study is to survey numerically comparison of two-phase and single-phase models of heat and mass transfer of Al2O3-water nanofluid liquid film flowing downward a vertical channel. A finite difference method is developed to produce the computational predictions for heat and mass transfer during the evaporation of the liquid film approached by the single-phase and two-phase models. The model solves the coupled governing equations in both nanofluid and gas phases together with the boundary and interfacial conditions. The systems of equations obtained by using an implicit finite difference method are solved by Tridiagonal Matrix Algorithm. The results show that the two-phase model is more realistic since it takes into account the thermophoresis and Brownian effects.
\end{abstract}

\section{Introduction}

The enhancement of heat transfer efficiency has been for decades the mean objective for heat exchangers manufacturer and research laboratories. Therefore, many active and passive solutions are being proposed. In this context, flowing liquid films have the advantage of higher heat transfer coefficient in comparison to single-phase flows. Flowing liquid films are largely described in the literature. Early Yan et al. [1] have experimentally analysed the evaporative cooling of a liquid film in a vertical channel with insulated walls. The results show a better cooling of the liquid film when low film flow rate and high inlet temperature were settled. The combined buoyancy effects on mass and heat transfer were examined by Feddaoui et al. [2]. They reported in their paper the best conditions to foster the heat transfer. Nait alla et al. [3, 4] investigated deeply the evaporation of alcohols and glycols inside fully and partially heated channels. Heated zones number and inlet conditions effects on the heat and mass transfer were discussed. Recently, Najim et al. [5] analysed the effect of salinity on the evaporation and heat exchange of a falling film inside a vertical tube. The authors reported the convenient boundary conditions for a better evaporation in desalination applications.

Nowadays, nanofluids seem to be a good passive solution. Plenty of problems are already investigated numerically as well as experimentally. However, those papers deal with problems containing one flowing fluid, and few papers devoted to phase change problems are restricted to droplets and motionless liquid films, see for instance $[6,7]$. The numerical examination of nanofluids is done either by the homogeneous model or by the twophase model. For example, Moumni et al. [8] studied the nanofluid effect on the mixed convection in lid-driven cavity. The used model assumes a uniform distribution of nanoparticles. Avramenko et al. [9] studied the nanoparticles effect in motionless film boiling. The analytical results showed that the increase of nanoparticles concentration promotes heat and mass transfer. Najim et al. [10] studied the effect of nanoparticles concentration on the heat and mass transfer during the evaporation of nanofluids inside a vertical channel. The authors claimed that nanoparticles presence improves the evaporation rate of the liquid film.

The main aim of this study is to predict heat and mass transfer characteristics by single phase (homogeneous fluid) and two-phase models in order to compare the two models. We are not aware of any published study on nanofluids effect on falling liquid film evaporation especially by the two-phase model excluding the study of Najim et al. [10]. The problem description and mathematical formulation are specified below.

\section{Mathematical modelling}

\subsection{Physical problem}

* Corresponding author: monssif.najim@edu.uiz.ac.ma 
Since the two flows take place in a downward direction the fields of velocity, pressure, temperature and concentrations at each section depend only on the upstream solutions. This property gives the parabolic character to the governing equations. Moreover, the difference between the wall and the liquid film interface temperatures is small. In consequence, the vapour diffusion is done at very small velocities, which allows neglecting the interface shear stress. For Reynolds number less than 1500, the liquid film flow may be considered laminar as quoted by Ueda and Tanaka [11].

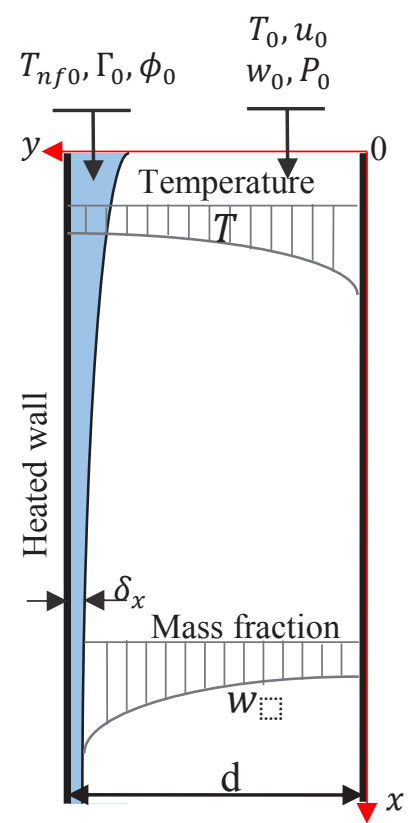

Fig. 1. The geometry of the channel with boundary conditions.

\subsection{Governing equations}

Proceeding with the above assumptions, the governing equations for laminar steady state flows are the continuity, momentum, energy species concentration and nanoparticles concentration equations. For the liquid phase those equations are:

$$
\begin{aligned}
& \nabla .\left(\rho_{n f} \vec{v}_{n f}\right)=0 \\
& \nabla \cdot\left(\rho_{n f} \vec{v}_{n f} \cdot \vec{v}_{n f}\right)=-\nabla P+\nabla \cdot\left(\mu_{n f} \nabla \vec{v}_{n f}\right)+\rho_{n f} \vec{g} \\
& \nabla \cdot\left(\rho_{n f} C_{p n f} T_{n f} \vec{v}_{n f}\right) \\
& =\nabla \cdot\left(\lambda_{n f} \nabla T_{n f}\right) \\
& +C_{p P} \rho_{P} \nabla \cdot\left(D_{B} \nabla \phi \nabla T_{n f}+D_{T} \frac{\nabla^{2} T_{n f}}{T_{n f}}\right) \\
& \nabla \cdot\left(\rho_{P} \phi \vec{v}\right)=\nabla \cdot\left(\rho_{P} D_{B} \nabla \phi+\rho_{P} D_{T} \frac{\nabla T_{n f}}{T_{n f}}\right)
\end{aligned}
$$

The gas flow governing equations are similar to the liquid film, thus, with the presence of the air, the gas flow is ternary and the equations may be written as:

$$
\begin{gathered}
\nabla \cdot\left(\rho_{G} \vec{v}_{G}\right)=0 \\
\nabla \cdot\left(\rho_{G} \vec{v}_{G} \cdot \vec{v}_{G}\right)=-\nabla P+\nabla \cdot\left(\mu_{G} \nabla \vec{v}_{G}\right)+\rho_{G} \vec{g}
\end{gathered}
$$

$$
\begin{aligned}
& \nabla \cdot\left(\rho_{G} \vec{u}_{G} C_{p G} T_{G}\right)=\nabla \cdot\left(\lambda_{G} \nabla T_{G}\right) \\
&+\nabla \cdot\left(\rho_{G}\left(C p_{a}-C p_{v}\right) D_{G m} \nabla w\right) \\
& \nabla \cdot\left(\rho_{G} \vec{u}_{G} w\right)=\nabla \cdot\left(\rho D_{G, i m} \nabla w\right)
\end{aligned}
$$

\subsection{Boundary conditions}

Boundary conditions are associated with the previous equations to solve the mathematical problem. Uniform velocity, temperature, mass fractions and nanoparticles concentration are specified at the channel inlet as follows:

- In liquid phase:

$$
\Gamma=\Gamma_{0} ; \quad T_{n f}=T_{n f 0}
$$

- In gas phase:

$$
u_{G}=u_{0} ; T_{G}=T_{0} ; P=P_{0} ; w=w_{0}
$$

- At the wet wall $(y=d)$

$$
\lambda_{n f} \frac{\partial T_{n f}}{\partial y}=q_{W} ; u_{n f}=0 ; D_{B} \frac{\partial \phi}{\partial y}=-\frac{D_{T}}{T_{n f}} \frac{\partial T_{n f}}{\partial y}
$$

- $\quad$ At the dry wall $(y=0)$

$$
\lambda_{G} \frac{\partial T_{G}}{\partial y}=0 ; u_{G}=0 ; \frac{\partial w}{\partial y}=0
$$

The matching conditions at the interface $\left(y=d-\delta_{x}\right)$ are:

$$
\begin{gathered}
u_{I}(x)=u_{G, I}=u_{n f, I} ; T_{I}(x)=T_{G}=T_{n f, I} \\
\tau_{I}=\left[\mu \frac{\partial u}{\partial y}\right]_{n f, I}=\left[\mu \frac{\partial u}{\partial y}\right]_{G, I} \\
{\left[\lambda \frac{\partial T}{\partial y}\right]_{n f, I}=\left[\lambda \frac{\partial T}{\partial y}\right]_{G, I}+\dot{m} h_{f g}}
\end{gathered}
$$

Where $h_{f g}$ is the latent heat of vaporization. The evaporating mass flux is:

$$
\begin{aligned}
& \dot{m}=-\frac{\rho_{G} D}{\left(1-w_{I}\right)} \frac{\partial w}{\partial y} \\
& D_{B} \frac{\partial \phi}{\partial y}=-\frac{D_{T}}{T_{n f}} \frac{\partial T_{n f}}{\partial y}
\end{aligned}
$$

\subsection{Nanofluids properties}

In this paper, we investigate the nanofluid of water containing the nanoparticles of Alumina $\mathrm{Al}_{2} \mathrm{O}_{3}$. To calculate the thermophysical properties of nanofluids the following equations are used:

$$
\begin{gathered}
\rho_{n f}=\phi \rho_{p}+(1-\phi) \rho_{L} \\
(\rho C p)_{n f}=\phi(\rho C p)_{n f}+(1-\phi)(\rho C p)_{L} \\
\lambda_{n f}=\lambda_{L}(1+7.47 \phi) \\
\mu_{n f}=\mu_{L}\left(1+39.11 \phi+533.9 \phi^{2}\right)
\end{gathered}
$$

\subsection{Solution method}

To solve governing equations, a fully implicit scheme is employed. The numerical solution is realized using finite 
difference method. Each finite-difference equation system forms a tridiagonal matrix, which can be solved using the TDMA method (Patankar [12]). It is still necessary to satisfy the global mass flow constraint. This is done by correcting the pressure gradient and axial velocity profile at each axial step, according to Raithby and Schneider method [13]. Moreover, the generated grid is nonuniform, in order to enhance numerical solutions accuracy.

\section{Results and discussion}

After the discretization of the governing equations combined with boundary conditions, calculations are performed for water-A12O3 nanofluid. The aim of this study is to compare the homogeneous model with the twophase model for computing the effect of nanoparticles on the heat and mass transfer. Details about the homogenous model applied to liquid film evaporation are given in [10]. To compare the two models several runs have been done for different conditions. The parameters of each run are described in the table below for 2 meters high and $2 \mathrm{~cm}$ wide channel and for a gas stream flowing at $\mathrm{Re}=2000$.

Table 1. Inlet and boundary conditions of each simulation run.

\begin{tabular}{|l|c|c|c|c|}
\hline & $\begin{array}{c}\boldsymbol{\Gamma}_{\mathbf{0}} \\
(\mathbf{k g} / \mathbf{m} . \mathbf{s})\end{array}$ & $\begin{array}{c}\boldsymbol{T}_{\mathbf{L 0}} \\
\left({ }^{\circ} \mathbf{C}\right)\end{array}$ & $\begin{array}{c}\boldsymbol{q}_{\boldsymbol{W}} \\
(\mathbf{W} / \mathbf{m})\end{array}$ & $\begin{array}{c}\boldsymbol{\phi}_{\mathbf{0}} \\
(\mathbf{\%})\end{array}$ \\
\hline Run 1 & 0.01 & 25 & 2000 & 2 \\
\hline Run 2 & 0.02 & 25 & 2000 & 2 \\
\hline Run 3 & 0.01 & 50 & 2000 & 2 \\
\hline Run 4 & 0.01 & 25 & 4000 & 2 \\
\hline Run 5 & 0.01 & 25 & 2000 & 4 \\
\hline
\end{tabular}

We present in figure 2 the evolution of interfacial latent heat along the channel for each model. The evaluation of boundary conditions effects on the predictions by the two models is done by considering run 1 as a reference. The latent heat flux increases for all cases by doubling the heat flux applied to the wall and by increasing the liquid film temperature, while it decreases by increasing the liquid film inlet flow rate. The increase of nanoparticles concentration fosters slightly the latent heat flux. It is clear that the latent heat predicted by the two models shows similar trend along the channel. However, the latent heat flux predicted under homogenous fluid hypothesis is underestimated and is always smaller than the two-phase model predictions. When doubling the heat flux applied at the wall (run 4), the difference between the two predictions increases, while when increasing the inlet liquid film temperature (run 3 ) the difference becomes very small. When doubling the concentration of the nanoparticles (run 5), the latent heat flux increases, but the two predictions keep the same differences as the reference case.

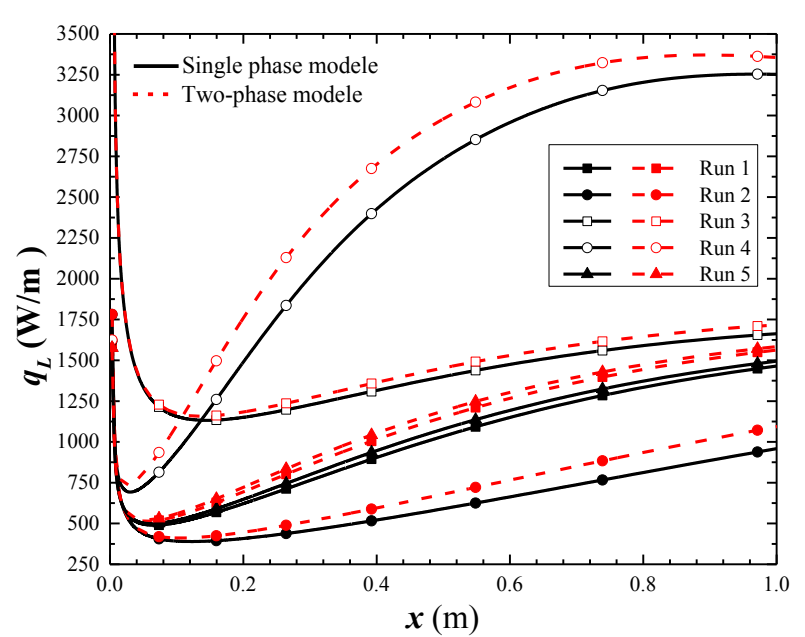

Fig. 2. Latent heat flux evolution along the channel.

Figure 3 demonstrates the sensible heat flux evolution along the channel as predicted by the two models. The sensible heat flux increases near the inlet to $\mathrm{x}=0.26 \mathrm{~m}$ for run 1 and 5 and to $\mathrm{x}=0.18 \mathrm{~m}$ for run 4 , and then decreases continuously to the channel outlet. For the highest inlet liquid film flow rate, the sensible heat flux increases along the channel while for the highest liquid film temperature q $\mathrm{S}$ decreases. It is obvious that the sensible heat flux predictions by the single-phase model are initially under those of the two-phase model for a given distance from the inlet, afterward they become above the two phase predictions. This distance is decreased from $0.65 \mathrm{~m}$ to $0.25 \mathrm{~m}$ by the increase of the heat flux at the wall. However, for the run 2 where the liquid film mass flow rate is doubled, the sensible heat flux predicted by twophase model is always larger than the single-phase predictions. At the channel outlet, the difference between the two predictions is very small except for run 2 . In fact, the homogeneous fluid hypothesis assumes that both base fluid and nanoparticles have the same properties in a given section. In reality, the nanoparticles do not keep a uniform distribution in the base fluid due to the thermophoresis and Brownian motions. In consequence, doubling the heat flux applied to the wall intensifies the thermophoretic force and the nanoparticles migrate from the hot zone (wall) to the colder zone (liquid film interface). In contrast, at high liquid film temperature (run 3 ) the liquid film approaches the saturation state, thus, the majority of the applied heat flux at wall is converted to latent heat flux. For this reason, the homogenous and twophase models predictions do not present a significant difference for high liquid film temperature. The inversion of the two models predictions order is due to the migration of nanoparticles. In fact, as the nanoparticles migrate to the interface, their concentration decreases near the hot zone. This leads to the decrease of the thermal conductivity near the wall. Consequently, less heat flux is transferred through the liquid film. This feature is not considered in the homogeneous model. 


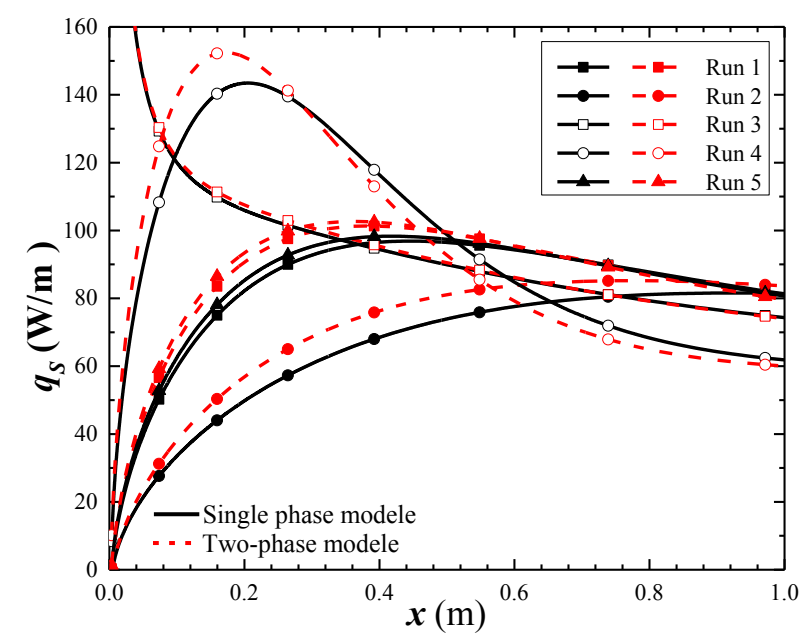

Fig. 3. Sensible heat flux distribution along the channel.

Figure 4 shows the evolution of accumulated evaporation rate along the channel. The results confirm the last findings and show that $M r$ increases along the channel. The evaporation is enhanced by doubling the wall heat flux and by increasing the liquid film temperature at the inlet. In contrast, the elevation of the liquid film mass flow rate decreases considerably the evaporation rate in comparison to the reference case. The accumulated evaporation rate evolution is very similar to the latent heat flux. Thus, higher difference between the two predictions is observed for high wall heat flux (run 4) while a very small difference is observed for high liquid film temperature. Those differences are due to the nanoparticles migration form the hot zone near the wall to the colder interface as already explained.

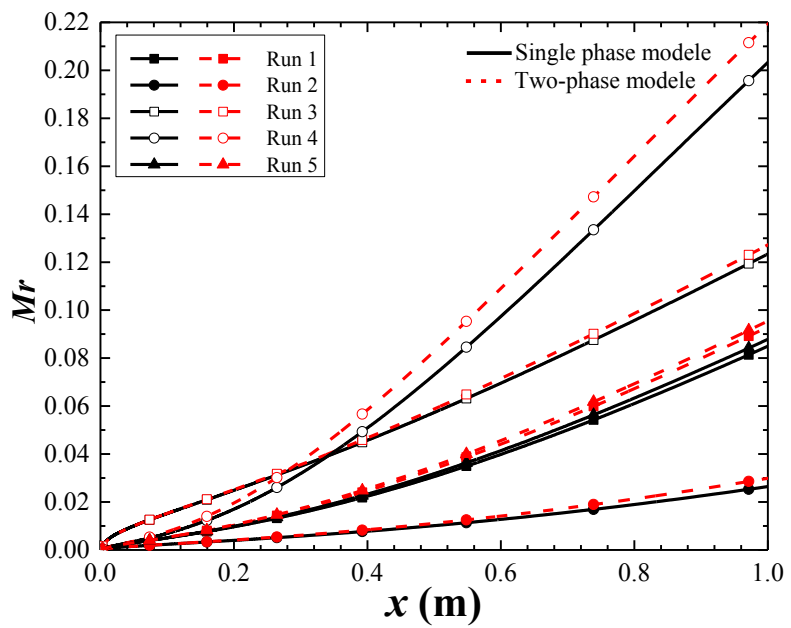

Fig. 4. Accumulated evaporation rate evolution along the channel.

Figures 5, 6, and 7, show the interfacial, gas bulk temperature and wall temperature along the channel. The plots indicate that those temperatures evolutions are very similar to the latent heat flux. As the liquid film flows down the channel, it absorbs the heat from the wall and its interfacial temperature increases. As the interface reaches the saturation condition, the evaporation begins and the gas flow absorbs the heat flux from the liquid film by the latent and sensible heat transfer modes. This leads to the increase of the gas flow temperature along the channel. Again, all values predicted by the single-phase model are underestimated and the lowest difference occurs for high inlet liquid film temperature, while the biggest difference is observed for higher wall heat flux and liquid film temperature.

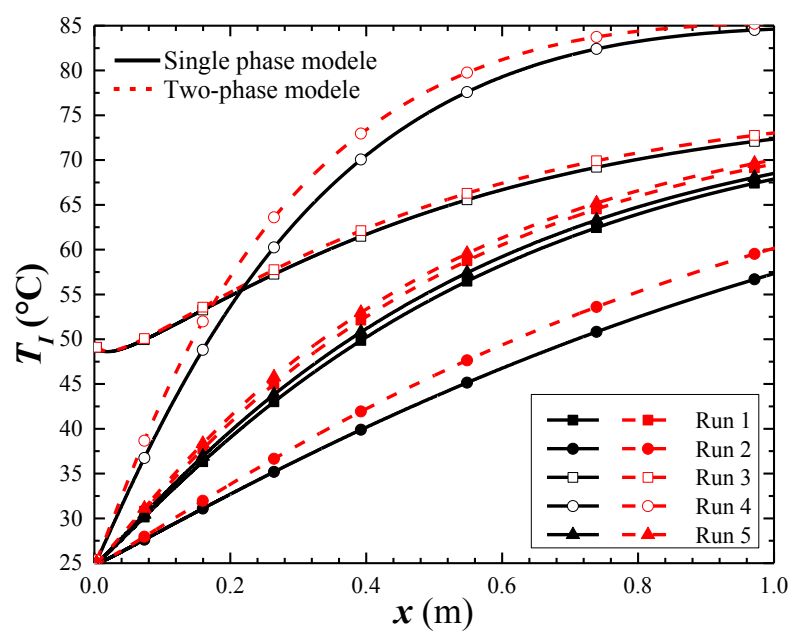

Fig. 5. Evolution of the interface temperature along the channel.

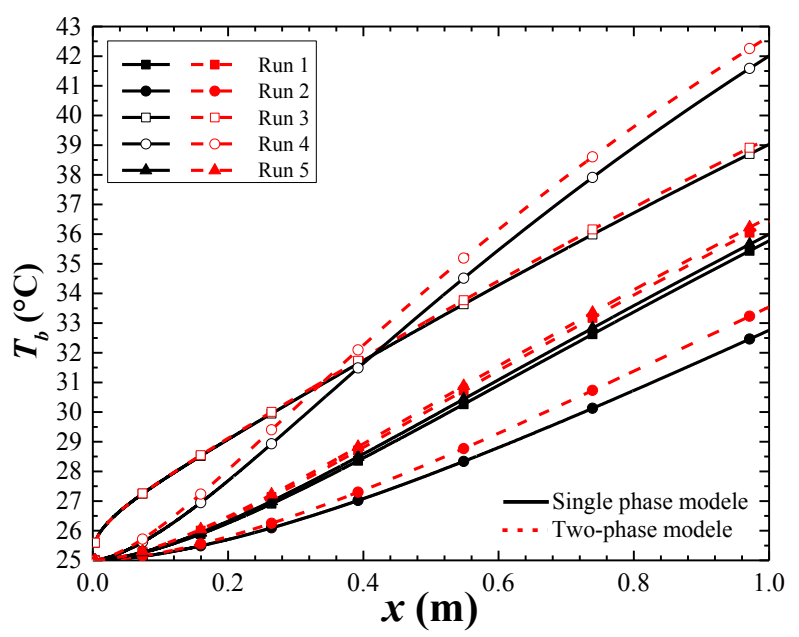

Fig. 6. Gas bulk temperature variation along the channel.

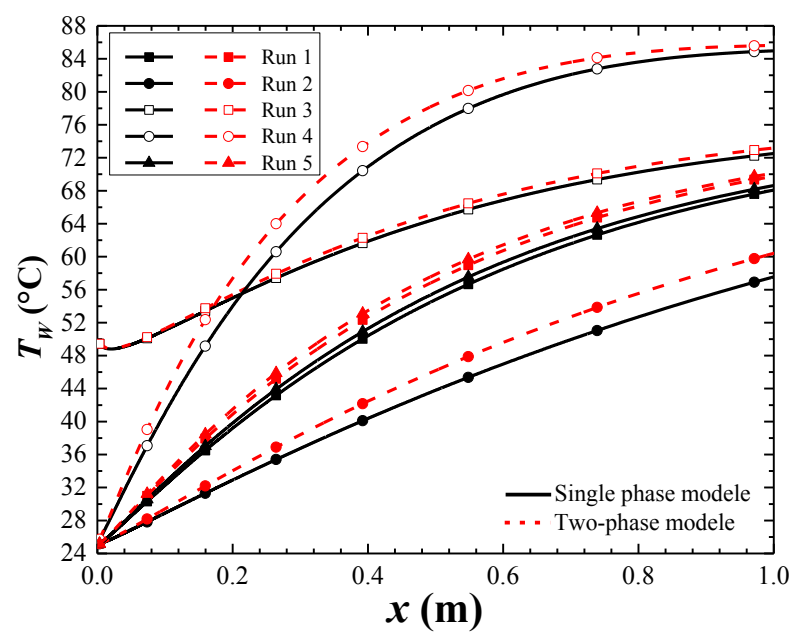

Fig. 7. Wall temperature evolution along the channel. 


\section{Conclusions}

The liquid Film evaporation inside a vertical channel with suspended $\mathrm{Al}_{2} \mathrm{O}_{3}$ nanoparticles is investigated numerically. The single phase (homogeneous fluid) and two-phase (base fluid+nanoparticles) models are used to run the simulations. Given that two-phase model considers the thermophoresis and Brownian motion, the interaction between base fluid and nanoparticles is computed and more realistic results were obtained. The results showed the existence of a difference between the two models predictions. However, for high liquid film temperature the difference is very small. The largest difference between the two models is observed for high wall heat flux and inlet liquid film flow rate. The singlephase model under-predict the results for all plotted parameters except for the sensible heat flux.

\section{Nomenclature}

$C_{p} \quad$ Specific heat, $\mathrm{J} \mathrm{kg}^{-1} \cdot \mathrm{K}^{-1}$

d Channel width, $\mathrm{m}$

D Mass diffusivity, $\mathrm{m}^{2} \cdot \mathrm{s}^{-1}$

$D_{B} \quad$ Brownian diffusion coefficient

$D_{T} \quad$ Thermophoretic diffusion coefficient

$\dot{\mathrm{m}} \quad$ Evaporation mass flux, $\mathrm{kg} \cdot \mathrm{s}^{-1} \mathrm{~m}^{-2}$

$\mathrm{Mr} \quad$ Mass Evaporation rate

$P \quad$ Pressure, $\mathrm{Pa}$

$q_{L} \quad$ Latent heat flux, W. $\mathrm{m}^{-2}$

$q_{S} \quad$ Sensible heat flux, W.m ${ }^{-2}$

$T \quad$ Temperature, $\mathrm{K}$

$u \quad$ Velocity component in $\mathrm{x}$ direction, $\mathrm{m} . \mathrm{s}-1$

$v \quad$ Velocity component in y direction, $\mathrm{m} . \mathrm{s}^{-1}$

$w \quad$ Vapour mass fraction

$x \quad$ Longitudinal coordinate

$y \quad$ Transversal coordinate

\section{References}

1. W.M Yan,TF. Lin,YL. Tsay, Int J Heat Mass Transf, 34, 1105-1111 (1991)

2. M. Feddaoui, A. Mir, E. Belahmidi, Int J Heat Mass Transf, 46, 3497-3509 (2003)

3. A. Nait Alla, M. Feddaoui, H. Meftah, Heat Mass Transf, 51, 1747-1760 (2015)

4. A. Nait Alla, M. Feddaoui, H. Meftah, Int J Heat Mass Transf, 89, 206-215 (2015)

5. M. Najim, M. Feddaoui, A. Charef, H. Meftah, Desalination, 408, 81-91 (2017)

6. K. Sefiane, R. Bennacer, Adv Colloid Interface Sci, 147-148, 263-271 (2009)

7. S. Vafaei, T. Borca-Tasciuc,MZ. Podowski, A. Purkayastha, G. Ramanath, PM. Ajayan, Nanotechnology, 17, 2523-2527 (2006)

8. H. Moumni, H. Welhezi, R. Djebali, E. Sediki, Appl Math Model, 39, 4164-4179 (2015).

9. A.A Avramenko, IV. Shevchuk, AI. Tyrinov, DG. Blinov, Int J Therm Sci, 92, 106-118 (2015)
10. M. Najim, M. Feddaoui, A. Nait Alla, A. Charef, Energy Procedia, 139, 122-127 (2017)

11. T. Ueda, T.,H. Tanaka, Int J Multiph Flow, 2, 261272 (1975)

12. S.V Patankar, Numerical heat transfer and fluid flow (Hemisphere/McGraw Hill, New York, 1980)

13. GD. Raithby, GE. Schneider, Numer Heat Transf, 2, 417-440 (1979) 